University of Wollongong

Research Online

Faculty of Science, Medicine and Health -

Papers: Part B

Faculty of Science, Medicine and Health

$1-1-2020$

\title{
Effectiveness of acupoint pressure on older people with constipation in nursing homes: A double-blind quasi-experimental study
}

\author{
Mu-Hsing Ho \\ University of Wollongong, mhh838@uowmail.edu.au \\ H.C. Chang \\ University of Wollongong, hchang@uow.edu.au \\ Megan F. Liu \\ Lin Yuan \\ University of Wollongong, lin_yuan@uow.edu.au \\ Jed Montayre
}

Follow this and additional works at: https://ro.uow.edu.au/smhpapers1

\section{Publication Details Citation}

Ho, M., Chang, H., Liu, M. F., Yuan, L., \& Montayre, J. (2020). Effectiveness of acupoint pressure on older people with constipation in nursing homes: A double-blind quasi-experimental study. Faculty of Science, Medicine and Health - Papers: Part B. Retrieved from https://ro.uow.edu.au/smhpapers1/1653

Research Online is the open access institutional repository for the University of Wollongong. For further information contact the UOW Library: research-pubs@uow.edu.au 


\title{
Effectiveness of acupoint pressure on older people with constipation in nursing homes: A double-blind quasi-experimental study
}

\begin{abstract}
Aims: To compare the differences between acupoint pressure, abdominal massage and laxatives in treatment of constipation for residents in two nursing homes. Background: There is lack of evidence on the utility of complementary therapies in the management of constipation among older adults. Design: Quasi-experimental. Methods: A total of 90 participants from two nursing homes in Taiwan were assigned to three groups: the control group (with laxatives only), group I (with laxatives and abdominal massage) and group II (with laxatives, acupoint pressure therapy [APT] and abdominal massage). The intervention was performed over 10 days. A double-blind technique was applied in both participants and the outcome assessors. Constipation as main outcome was evaluated and recorded for 10 days. Observation of the frequency of defecation and the consistency, particularly firmness of faeces over each 24 hours' period was recorded. The Transparent Reporting of Evaluations with Nonrandomized Designs (TREND) checklist was utilised in reporting methods and findings. Results: Three variations were revealed as interface factors and showed significant differences in each group. The results showed a statistically significant decrease in constipation in the experimental groups I and II. The scores of defecation frequency, difficulty degree and time of defecation, stool quality and awareness of defecation were obviously improved after treatment. Conclusions: We concluded APT could be used in conjunction with laxatives and must be considered as a long-term intervention. The combination of APT, abdominal massage and laxatives is superior to both abdominal massage with laxatives and laxatives alone. Relevance to clinical practice: Acupoint pressure as an effective complementary therapy of constipation among older adults living in nursing home provides a non-pharmacological, independent nursing intervention that nurses could use. This is relevant to nursing home settings where nurses make autonomous decision on important clinical assessments and interventions.
\end{abstract}

\section{Keywords}

double-blind, homes:, nursing, effectiveness, acupoint, pressure, older, people, constipation, study, quasiexperimental

\section{Publication Details}

Ho, M., Chang, H. C. R., Liu, M. F., Yuan, L. \& Montayre, J. (2020). Effectiveness of acupoint pressure on older people with constipation in nursing homes: A double-blind quasi-experimental study. Contemporary Nurse, online first 1-19. 
Publisher: Taylor \& Francis \& Informa UK Limited, trading as Taylor \& Francis Group Journal: Contemporary Nurse

DOI: $10.1080 / 10376178.2020 .1813042$

Running title: ACUPOINT PRESSURE THERAPY

Effectiveness of acupoint pressure on older people with constipation in nursing homes:

\section{A double-blind quasi-experimental study}

by

Mu-Hsing Ho ${ }^{1}$, Hui Chen (Rita) Chang ${ }^{2}$, Megan F. Liu ${ }^{3}$, Lin Yuan ${ }^{4}$, Jed Montayre ${ }^{5}$

${ }^{1} \mathrm{MSc}, \mathrm{RN}, \mathrm{PhD}$ student, School of Nursing, Faculty of Science, Medicine and Health, University of Wollongong, New South Wales, Australia and Registered Nurse, Department of Nursing, Taipei Medical University Hospital, Taipei, Taiwan.

E-mail:mhh838@uowmail.edu.au

Address: B41, R116, Northfields Avenue, University of Wollongong NSW 2522

ORCID iD: https://orcid.org/0000-0002-9443-4082

${ }^{2} \mathrm{PhD}, \mathrm{RN}$, Senior Lecturer, School of Nursing, Faculty of Science, Medicine and Health, University of Wollongong, New South Wales, Australia

E-mail: hchang@uow.edu.au

ORCID iD: https://orcid.org/0000-0002-8305-0585

${ }^{3} \mathrm{PhD}, \mathrm{RN}$, Associate Professor, School of Gerontology Health Management, Taipei Medical University, Taipei, Taiwan

E-mail: fangliu@tmu.edu.tw

Address: 250 Wuxing St., Taipei, Taiwan, 110

ORCID iD: https://orcid.org/0000-0001-5942-8659

${ }^{4} \mathrm{PhD}$, Research Staff, School of Nursing, Faculty of Science, Medicine and Health,

University of Wollongong, New South Wales, Australia

E-mail: yuan_lin@uow.edu.au

Address: B41, Northfields Avenue, University of Wollongong NSW 2522

${ }^{5} \mathrm{PhD}, \mathrm{RN}$, Senior Lecturer, School of Nursing and Midwifery, Western Sydney University,

New South Wales, Australia

E-mail: j.montayre@westernsydney.edu.au

Address: Campbelltown Campus, Buidling 7.G.11 Locked Bag 1797 Penrith NSW 2751

Australia

ORCID iD: https://orcid.org/0000-0002-2435-8061

Corresponding author: Hui Chen (Rita) Chang, $\mathrm{RN}, \mathrm{PhD}$

School of Nursing, University of Wollongong

Address: B41, R111, Northfields Avenue, University of Wollongong NSW 2522 
Tel: +61242213174

Fax: + 61242213137

E-mail: hchang@uow.edu.au

\section{Conflict of interest statement}

The authors declare that they have no conflicts of interest concerning this article.

\section{Funding}

This research was conducted without any funding or grant aid from any funding agency in the public, commercial or not-for-profit sectors. 


\title{
Effectiveness of acupoint pressure on older people with constipation in nursing homes:
}

\section{A double-blind quasi-experimental study}

\begin{abstract}
Aims: To compare the differences between acupoint pressure, abdominal massage and laxatives in treatment of constipation for residents in two nursing homes.

Background: There is lack of evidence on the utility of complementary therapies in the management of constipation among older adults.

Design: Quasi-experimental.

Methods: A total of 90 participants from two nursing homes in Taiwan were assigned to three groups: the control group (with laxatives only), group I (with laxatives and abdominal massage) and group II (with laxatives, acupoint pressure therapy [APT] and abdominal massage). The intervention was performed over 10 days. A double-blind technique was applied in both participants and the outcome assessors. Constipation as main outcome was evaluated and recorded for 10 days. Observation of the frequency of defecation and the consistency, particularly firmness of faeces over each 24 hours period was recorded. The Transparent Reporting of Evaluations with Nonrandomized Designs (TREND) checklist was utilised in reporting methods and findings.

Results: Three variations were revealed as interface factors and showed significant differences in each group. The results showed a statistically significant decrease in constipation in the experimental groups I and II. The scores of defecation frequency, difficulty degree and time of defecation, stool quality and awareness of defecation were obviously improved after treatment.
\end{abstract}

Conclusions: We concluded APT could be used in conjunction with laxatives and must be considered as a long-term intervention. The combination of APT, abdominal massage and laxatives is superior to both abdominal massage with laxatives and laxatives alone.

Relevance to clinical practice: Acupoint pressure as an effective complementary therapy of constipation among older adults living in nursing home provides a non-pharmacological, independent nursing intervention that nurses could use. This is relevant to nursing home settings where nurses make autonomous decision on important clinical assessments and interventions.

Key words: constipation, acupressure, alternative therapy, older people, long-term care 


\section{Introduction}

Constipation is a common condition occurring in older adults, which is defined as difficult stool passage and stool frequency of less than three bowel movements per week (Rao \& Go 2010; Rughwani, Nakagawa, \& Shah, 2014). The estimate prevalence of constipation is $33.5 \%$, particularly in older people aged over 60 years (Forootan, Bagheri, \& Darvishi, 2018; GallegosOrozco, Foxx-Orenstein, Sterler, \& Stoa, 2012). Moreover, constipation is a very common condition in residential aged care facilities. A Finnish study reported that $79 \%$ to $81 \%$ of the residents in long-term care facilities and nursing homes suffer from constipation (Kinnunen, 1991). In the United States about $85 \%$ of patients with constipation that require medical treatment were already using laxatives. It is estimated that 82 million US dollars are spent on over-the-counter laxatives (Menees, et al. 2015). This spending was towards middle aged and older people, who commonly develop constipation because of the age-related physiological changes in digestive and metabolic systems. Within the Taiwanese context, it can be argued that there were known cross-cultural differences between Asian and some western countries in terms of dietary intake (Prescott, Young, O'Neill, Yau, \& Stevens, 2002; Smith, Knight, Sahota, Kernohan, \& Baker, 1993). The use of traditional, herbal and complementary medicine in treating common health conditions have been popular in some Asian cultures (Hsiao, et al., 2006; Lim, Sadarangani, Chan, \& Heng, 2005). This article presents the findings on the use of complementary therapy for constipation among older populations in institutionalised settings.

\section{Background}

Constipation is more of symptom than a disease process, which creates discomfort and impacts on other conditions' clinical presentations. Currently, constipation is generally managed using laxatives and lifestyle modifications, both of which have presented contradictory findings regarding their effectiveness (Brown \& Lawrie, 2006; Wald, et al, 2007). However, long-term use of laxatives can cause shrinking of intestinal smooth muscle resulting in the patient's loss of bowel movement sensation, leading to faecal impaction which causes overextension of the reetum. Evidence also suggested that overuse of laxatives or enemas/suppositories will result in constipation (Chattri, 2018; Seifert, 2019).

From a nursing perspective, the priority interventions included rebuilding bowel habits by promoting increased physical activity, fibre and fluid intake. These interventions were based on the science of physiological peristalsis and water reabsorption together with digestive 
processes. However, these interventions are not always effective (Towers, et al., 1994). On the other hand, there are non-traumatic and complementary therapies identified, such as auriculotherapy, behavioural therapy, and reflexology. However, these alternative therapies have seldom been used or found to be effective in the treatment of constipation (Richmond, 2003). There is very little evidence documented on complementary therapies. For example, one earlier randomized controlled trial found out that abdominal massage therapy decreased the severity of constipation and increased bowel movement after 8 weeks, but did not lead to a decrease in laxative intake (Chen, et al., 1998).

Currently, acupoint pressure therapy (APT) applied to a variety of symptoms has been documented in literature more frequently. In Taiwan, the Executive Department of Health issued a directive to enable trained registered nurses (RNs) to use acupuncture point pressure to improve some clinical conditions such as digestive function and quality of sleep (Mehta, Dhapte, Kadam, \& Dhapte, 2017; Wu \& Lin, 2003). APT is the application of pressure to specific acupoints on the body and is believed to open up the flow of $Q i$, the energy that restores balance within the human body (Liu, 1988). APT not only has significant effect in treatment of vomiting caused by chemotherapy but also promotes intestinal peristalsis in pregnancy and postpartum caesarean section patients (Shin \& Park, 2016) and quality of recovery among postoperative patient (Noll, et al., 2019). Previous studies reported promising results in managing constipation using acupoint pressure though results were found inconsistent (Lin, 2011).

APT is readily accepted in clinical practice because it is safe and convenient to apply with positive therapeutic outcomes. However, selection of appropriate acupoint sites is an important clinical consideration that needs to be undertaken with caution and should be consulted with health professionals, particularly if the patient has serious health conditions (Mehta, et.al., 2017; Zhang, et al., 2001). The underlying principle behind APT in managing constipation is the regulation of the stomach and large intestinal activity, as well as $Q i$ flow and blood circulation which results in an increased intestinal peristalsis, thus generating the need for defecation (Freeman \& Lawlis, 2001). However, the mechanism of action for APT is unknown and there is limited evidence of the effectiveness of acupressure in treating constipation among older people in nursing homes. Previous Chinese studies using auricular acupressure for managing constipation showed no clear evidence of effectiveness due to methodological limitations such as small sample size. (Zhang, et al., 2006). Therefore, we conducted this study to evaluate the 
complementary effects of APT in relieving constipation symptoms among older people in nursing homes in Taiwan. This study aimed to compare the effect of acupoint pressure, abdominal massage and use of laxatives for constipation in nursing home residents.

\section{Methods}

\section{Research design and participants}

In this study, the double blind controlled trial was designed with pre-test and post-test methods in three groups. The participants were recruited from two nursing homes in the Hsinchu district in Taiwan. The inclusion criteria recruited residents who: a) had fewer than 2 bowel movements per week within a month of the study; b) those who voluntarily participated and completed the consent form; c) did not have abdominal surgery in the past 6 months; d) without abdominal tumours or any complaints of pain. Ninety participants met the above criteria. The participants were assigned to either the experimental or the control group by principal researcher according to the location of the unit in nursing homes. The principal researcher allocated participants individually and based on the sequential order when participant consent was obtained. In this study, the assessor RN who was responsible for data collection was blinded to the participants' group assignments and the intervention they received. In this study, time series design methods involving three groups were adopted. The number of defecations and stool softness and hardness were observed daily for 11 days from June 15, 2015 to June 25, 2015. The Transparent Reporting of Evaluations with Nonrandomized Designs (TREND) statement checklist was performed to report the methodology in this quasi-experimental study (Des Jarlais, Lyles, Crepaz, \& Trend Group, 2004) (See Supplementary File 1).

\section{Intervention}

In order to explore the effect of APT and abdominal massage on the improvement of constipation, residents who met the criteria were assigned to two experimental groups - (I) laxatives and abdominal massage and (II) laxatives, acupoint pressure and abdominal massage and control group using laxatives only. Common pharmacological treatment for constipation uses laxative agents. Therefore, all participants received laxatives as routine treatment. Participants in the control group received laxatives only. Abdominal massage is a common non-pharmacological intervention and acupoint pressure is an approved nursing intervention for constipation. Abdominal massage and acupoint pressure were selected for experimental treatment because of their mostly positive non-invasive effect, and ability to be performed at 
any time. Participants in both experimental groups received the abdominal massage method performed by a trained RN applying downward pressure to about $3-5 \mathrm{~cm}$ with continuous stimulation over ten minutes each time for ten days. They were trained according to a standard guideline or protocol for practice (Preece, 2002) including the consistency of the pressing techniques and timing. A training workshop was conducted by an experienced Chinese medical practitioner who had performed acupressure for 10 years. The workshop was designed to equip two RNs with APT, abdominal massage, and group supervision skills.

Five acupoints, namely Tianshu (ST25), Zhongdu (GB32), Shenmen (TF4), Zusanli (ST36) and Sanyinjiao (SP6) were selected for experimental group II because of their positive effects in relieving constipation symptoms (World Health Organization, 1993; Yan et al., 2005). These acupoint areas have systemic physiological effects involving upper and lower extremities. For a more comprehensive information please see the Standard acupuncture nomenclature: a brief explanation of 361 classical acupuncture point names and their multilingual comparative list. published by WHO (https://apps.who.int/iris/handle/10665/207716). Trained RNs pressed three to five centimetres depth into each acupoint lasting for approximately $1 \mathrm{~min}$, once a day for total 10 days. All participants were assessed prior to the day of intervention using the constipation defined features observation questionnaire scale. The frequency of defecation and consistency, particularly firmness of faeces were recorded. From the next day, intervention measures were taken for 10 consecutive days with observing defecation patterns and were recorded daily.

\section{Measurements}

Participants' demographic and clinical profiles were collected before administering the intervention. Constipation symptoms were measured by constipation defined features observation questionnaire. This questionnaire was developed by researchers on the basis of the NANDA-I nursing diagnosis of constipation, other research results and clinical experience. The internal consistency and test-retest reliability were reported to be high (Cronbach's [alpha] $=0.89$ and intra-class correlation $=0.75$ ). It contains 28 items for patient self-completion to rate ease of defecation, frequency and consistency, firmness of faeces, feeling of incomplete bowel evacuation, and others signs and symptoms of constipation. Participants were asked to rate each item on a 3 -point Likert scale: $0=$ no problem, $1=$ some problems, and $2=$ severe problems with an overall score ranging from 0 to 56 (where higher scores indicate more severe symptoms or a more severe adverse effect of constipation). 
Observations were made by trained RNs. Acupoint pressure and abdominal massage record sheets were designed by the researchers to record the intervention date, execution time, the frequency of defecation, the consistency, firmness of faeces, the nature of faeces, physical activity, and dietary intake of fruit and vegetables on the day before the intervention, for 10 consecutive days.

\section{Procedures}

First, ethical approval was obtained from the University ethics committee with the reference NUR576891_14. In this study, a double-blind technique was adopted. The assessor was an RN from the nursing home. The $\mathrm{RN}$ was trained, according to the required observation questionnaire in recording the consistency of stools. For an accurate doeumentation process, trial assessment and recordings were undertaken by the $\mathrm{RN}$ for 10 times prior to the intervention. Both the participants and the assessor $\mathrm{RN}$ who was responsible for data collection were blinded to the participants' group assignments. In obtaining consent, participants were explained and had fully understood that they will receive abdominal massage, APT and laxatives in non-specific order when to receive these and were unaware of their group allocation. After data collection, all participants except experimental group II received abdominal massage and APT. This was undertaken to ensure that ethical considerations were employed and that data collection for the purpose of the study was conducted in specific time points and did not disadvantage any participant.

The aims and procedure of the study were explained to the participants who met the study criteria and agreed to participate. They provided written consent. Participants would be discontinued from the study if they became physically ill, experienced discomfort during the intervention, or if they failed to participate in $90 \%$ of the total intervention. The participants were assessed by interviews, observation, physical examinations, and reference to medical records to assess who met the criteria. Participants who were discharged, transferred, died or were otherwise unable to continue to receive the massage and APT were replaced by other eligible residents. Participants were expected to maintain usual dietary habits and levels of physical activity.

\section{Statistical analysis}

$\mathrm{G}^{*}$ power 3.1.9.2 software was used to conduct sample size calculation. First, the model setting based on the statistics test is $\mathrm{F}$ tests with linear multiple regression: fixed model, $R^{2}$ deviation 
from zero. We calculated the sample size by giving the following parameters: Effect size $f^{2}=0.25$, alpha $=0.05$, Power $(1$-beta $)=80 \%$, the estimate sample size calculation by $\mathrm{G}^{*}$ power is 82 .

Statistical Package for Social Sciences (Version 18.0), EPI-INFO and SAS statistical software were used. One-way ANOVA and chi-squared tests were used for comparisons of the baseline characteristics among the three study groups. The homogeneity of the three study groups was assessed by comparing their baseline outcome scores and socio-demographic characteristics using the Chi-square test (for nominal variables) and the independent sample t-test or MannWhitney U test (for continuous variables). The Generalized Estimating Equations (GEE) model can account for intra-correlated repeated measure's data and accommodate missing data caused by dropout, provided the data are missing at completely random (Singer, 2003) and thus is particularly suitable for intention-to-treat analysis without the need of imputation. GEE model also aimed to assess the differential change of the outcome at each post-test time point relative to the baseline between the underlying comparison groups. Therefore, the GEE model was adopted to examine the complementary effects of APT in managing constipation in this study. For outcome measures showing significant results in the GEE test, subgroup analyses were performed to examine any significant differences in mean scores between subgroups for gender, age, use of laxatives, amount of dietary fibre, physical activity, and chronic disease. All statistical tests involved were 2 -sided and a $p$-value of $<0.05$ was considered statistically significant.

\section{Results}

\section{Characteristics of participants}

One hundred and ten participants from two nursing homes were recruited over a month and allocated into either two experimental groups and one control group ( $\mathrm{n}=35-36$ in each group). Twelve participants have withdrawn participation because of diarrhoea prior to the intervention and eight discontinued from the study before receiving intervention because of feeling uncertain about the intervention. With 28 and 32 participants of the experiment groups I and II respectively completed the intervention and the 10 days' follow-up measures. Thirty participants in the control group completed their 10 days of measures using laxatives only (see Table 1). Intention-to-treat analysis strategy was used in this study.

Homogeneous test of the baseline characteristics using the conventional $p$-value of $<0.05$ 
between control and experimental groups showed: three variations as interference factors were turned out and there was no relevant evidence from each of the groups. Three potential control variables were identified, namely dietary intake of fruit, fluid intake and presence of chronic disease (Table 2).

\section{Effectiveness of acupoint pressure therapy and abdominal massage}

The experimental groups I and II on the constipation defined features observation scores were significantly lower compared with those of the control group $(F=24.027, p<0.001)$. However, there was no difference between the experimental group I and control group. The results indicated that the application of APT and abdominal massage had an effect on constipation measurements and abdominal massage alone did not.

The intervention may be affected by these three factors (1) fluid intake: fluid intake in the control group is significantly higher than that in the experimental groups. The more fluid intake, the more frequent the defecation can be, which may be one of the factors affecting the impact of intervention (2) fruit intake: $71.5 \%$ and $40.6 \%$ of participants in experimental groups I and II respectively had 2 serves of fruit per day, while $43.3 \%$ in control group had 2 serves of fruit per day, indicating the number of defecations in the experimental group I should be more than other groups. It can be seen that this interference factor is inconsistent with the research hypothesis that with less intake of vegetables and fruit, the severity of constipation is greater. (3) presence of chronic disease: considering that constipation in most cases is a symptom of underlying chronic conditions. There were fewer participants with chronic diseases in the experimental group II than the other two groups (18.7\%), indicating that this group should be less constipated, while $36.7 \%$ of the control group and $35.7 \%$ of the experimental group had chronic diseases indicating the participants in the experimental group II may be less prone to constipation. It can be seen that this interference factor is relevant in affecting the outcome of abdominal massage or APT.

With adjustment of the eight potential covariates (age, bowel movements less than twice a week, bedridden, activity dependent, oral intake methods, and the three control variables fruit, fluid intake and chronic disease in the GEE model, the complementary effects of APT on the constipation symptoms are summarized in Table 3. Because there are three different control variables among the groups, these three variables become interference factors (chronic disease and fluid and fruit intake) and are statistically controlled to compare the effects. Table 3 shows 
the effectiveness of interventions after controlling the interference factors and significant differences between the groups $(p<0.04-0.001)$.

An overall improvement was found in constipation symptoms and the frequency of bowel movements on completion of intervention at 10th-day follow-up time, relative to the baseline in experimental groups. Most importantly, the experimental group II demonstrated the greatest improvement in both constipation symptoms and frequency of bowel movements on completion of APT at 10th-day follow-up time among the three study groups.

\section{Discussion}

This is the first known interventional study to evaluate the complementary effects of APT in relieving constipation symptoms among nursing home residents in Taiwan. There is heterogeneity of participants' baseline measurements across the study groups. However, in this study, all participants took regular laxatives. Apparently, laxatives were not effective in relieving participants' constipation symptoms. Such findings are similar to that of a previous study in which $23-45 \%$ of patients were constipated even though they were taking laxatives (Brown \& Lawrie, 2006).

This study has produced evidence of APT and abdominal massage effects for constipation and is statistically significant and clinically meaningful. In a study of older people with severe constipation, Lin et al. (2011) used a similar treatment protocol (an acupressure program with abdominal massage) and a similar primary outcome (i.e., symptom severity). They reported a substantial effect on symptom reduction (effect size $f^{2}=3.03$ ) immediately after the acupressure intervention. In the present study, however there was no difference between the experimental group I and II. This indicates the APT alone may not have impact on the constipation. This differs from results of previous studies using complementary and alternative therapies (Jung \& Jun, 2008; Kim, Kang, \& Byun, 2014; Park, et al., 2011). However, one study that assessed the effects of abdominal massage and meridian acupressure reported no significant difference in constipation symptoms between the experimental and control groups (Yoon, et al., 2014). This might have resulted from insufficient treatment time. Insufficient compression time to stimulate acupoint sites may have affected the outcome.

The results of this study inform evidence-based practice for nurses who have skills and training with acupoint intervention. Acupoint pressure as an effective complementary therapy of constipation among older adults living in nursing home provides a non-pharmacological, 
independent nursing interventions that nurses can use. This is relevant to nursing home settings where nurses make autonomous decision on important clinical assessments and interventions. Additionally, acupressure is a user-friendly treatment, individuals living with chronic illnesses yet are physically capable to apply pressure to acupoint sites could easily adopt and implement this intervention using a simple treatment protocol to relieve their symptoms and prevent common uncomfortable experiences from acute or long-term constipation.

\section{Limitations and recommendations}

Firstly, in the present study, the potential interference factors were controlled in the statistical analysis. The results indicate the valuable effect of APT on constipation, but because of the differences in the number of participating residents from the nursing homes it might cause an unbalanced representation in each study arms. The randomisation was difficult to perform in the actual research settings as we only recruited two nursing homes. It is recommended that similar studies with randomised controlled trial design need to be done in the future. In order to minimise interference factors, before the data collection, fluid and fruit intake as well as the history of chronic illness or existing medical conditions need to be controlled as far as possible (Forootan, et al., 2018).

Secondly, due to time and funding limitation, the intervention period was time-limited to 10 days and there was no follow-up undertaken. Therefore, it is suggested that future studies should examine the frequency and time of acupressure therapy and a longer follow-up period to ensure sustainability and clinical efficacy in the treatment of constipation. Service users' preference for and satisfaction with the APT regime should also be considered in future research.

Lastly, one limitation is with the sample size. The sample size is modest, as fewer than 90 participants completed the study and were of advanced age groups within the older adult population groups (mean $=76.82$ ). Therefore, generalization of the current findings should be made with caution. To overcome these limitations, larger sample size is recommended in further clinical trials.

\section{Conclusion}

There has been no controlled trial on the effects of APT among nursing home residents in Taiwan. The findings of the current study provided encouraging evidence to support the clinical 
value of APT and abdominal massage in managing constipation.

\section{Relevance to clinical practice}

The findings from the current study suggest that trained health care professionals can effectively integrate APT within nursing home settings. Nurses will be able to evaluate their current management of constipation among older adults living in nursing home in consideration of APT as a potential intervention. Furthermore, the study methodology suggests good reference for further clinical studies in adopting APT as an intervention in the local context especially when there is a lack of clinical trials in this area. However, in nursing practice, there is a need to implement this intervention with caution and only trained professionals should apply this intervention. It would be useful to conduct further research on the self-help approach to acupressure adopted in this trial over a longer follow-up period and in different practice settings. This would allow the investigation of whether efficacy in illness self-management can be enhanced in a range of older people with constipation.

\section{References}

Brown, L., \& Lawrie, I. (2006). Constipation: patient perceptions compared to diagnostic tool. Palliative Medicine, 20, 717-718.

Chattri, G. L. (2018). Laxatives. In Nursing Drug Dosages and their Calculations (pp. 179181). jaypee.

Chen, L. L., Wang, C. J., \& Cai, X. Z. (1998). Explore on the effects of acupoint pressure in the improvement of peristalsis after caesarean. Nursing Study, 6(6), 526-534.

Des Jarlais, D. C., Lyles, C., Crepaz, N., \& Trend Group. (2004). Improving the reporting quality of nonrandomized evaluations of behavioral and public health interventions: the TREND statement. American journal of public health, 94(3), 361-366.

Forootan, M., Bagheri, N., \& Darvishi, M. (2018). Chronic constipation: A review of literature. Medicine, 97(20), e10631. doi: 10.1097/MD.0000000000010631

Freeman, L. W., \& Lawlis, G. F. (2001). Incomplementary and alternative therapy: a research-based approach. St. Louis: Mosby.

Gallegos-Orozco, J. F., Foxx-Orenstein, A. E., Sterler, S. M., \& Stoa, J. M. (2012). Chronic constipation in the elderly. The American Journal of Gastroenterology, 107, 18-25.

Hsiao, A. F., Wong, M. D., Goldstein, M. S., Becerra, L. S., Cheng, E. M., \& Wenger, N. S. (2006). Complementary and alternative medicine use among Asian-American 
subgroups: prevalence, predictors, and lack of relationship to acculturation and access to conventional health care. Journal of Alternative and Complementary Medicine, 12(10), 1003-1010. doi: 10.1089/acm.2006.12.1003

Jung, Y. H., \& Jun, J. Y. (2008). The effect of meridian acupressure on constipation in the bedridden aged with stroke. Korean Journal of Adult Nursing, 20, 664-673.

Kim, M. Y., Kang, E. H., \& Byun, E. K. (2014). Effects of meridian acupressure on constipation in the institutionalized elderly. The Korean Journal of Rehabilitation Nursing, 17, 48-55. doi:10.7587/kjrehn.2014.48

Kinnunen, O. (1991). Study of constipation in a geriatric hospital, day hospital, older people's home and at home. Aging, 3, 161-170.

Lim, M. K., Sadarangani, P., Chan, H. L., \& Heng, J. Y. (2005). Complementary and alternative medicine use in multiracial Singapore. Complementary Therapies in Medicine, 13(1), 16-24. Doi: 10.1016/j.ctim.2004.11.002

Lin, S. F., Tang, W. R., Chang, Y. C., \& Maa, S. H. (2011). Effectiveness of acupressure on elderly patients with constipation. Journal of Nursing and Healthcare Research, 7(3), 175-187.

Liu, Y. (1988). The essential book of traditional Chinese medicine. New York: Columbia University Press.

Mehta, P., Dhapte, V., Kadam, S., \& Dhapte, V. (2017). Contemporary acupressure therapy: Adroit cure for painless recovery of therapeutic ailments. Journal of traditional and complementary medicine, 7(2), 251-263.

Menees, S. B., Guentner, A., Chey, S. W., Saad, R., \& Chey, W. D. (2015). How do US gastroenterologists use over-the-counter and prescription medications in patients with gastroesophageal reflux and chronic constipation? The American Journal of Gastroenterology, 110(11), 1516-1525.

Noll, E., Shodhan, S., Romeiser, J. L., Madariaga, M. C., Page, C., Santangelo, D., ... \& Bennett-Guerrero, E. (2019). A randomised controlled pragmatic trial of acupressure therapy on quality of recovery after surgery. European journal of anaesthesiology.

Park, J. E., Sul, J. U., Kang, K., Shin, B. C., Hong, K. E., \& Choi, S. M. (2011). The effectiveness of moxibustion for the treatment of functional constipation: a randomized, sham-controlled, patient blinded, pilot clinical trial. $B M C$ Complementary and Alternative Medicine, 11, 124. doi:10.1186/1472-6882-11-124

Preece, J. (2002). Introducing abdominal massage in palliative care for the reflief of constipation. Complementary Therapies in Nursing and Midwifery, 8(2), 101-105. 
Prescott, J., Young, O., O'Neill, L., Yau, N. J. N., \& Stevens, R. (2002). Motives for food choice: a comparison of consumers from Japan, Taiwan, Malaysia and New Zealand. Food Quality and Preference, 13(7), 489-495. doi: 10.1016/S0950-3293(02)00010-1

Rao, S. S., \& Go, J. T. (2010). Update on the management of constipation in the elderly: new treatment options. Clinical Interventions in Aging, 5, 163-171. doi: 10.2147/cia.s8100

Richmond, J. (2003). Prevent through of constipation risk management. Nursing Standard, 17(16), 39-48.

Rughwani, N., Nakagawa, S., \& Shah, B. (2014). Constipation in the long-term care setting. Journal of Case Management: The journal of long term home health care, 15(2), 8390. doi: 10.1891/1521-0987.15.2.83

Seifert, R. (2019). Drugs for Treatment of Gastrointestinal Diseases. In Basic Knowledge of Pharmacology (pp. 167-180). Springer, Cham.

Shin, J., \& Park, H. (2016). Effects of auricular acupressure on constipation in patients with breast cancer receiving chemotherapy: a randomized control trial. Western Journal of Nursing Research, 40(1), 67-83. doi:10.1177/0193945916680362

Singer, J. D., \& Willett, J. B. (2003). Applied longitudinal data analysis: modelling change and event occurrence. New York: Oxford University Press.

Smith, Z., Knight, T., Sahota, P., Kernohan, E., \& Baker, M. (1993), Dietary patterns in Asian and Caucasian men in Bradford: differences and implications for nutrition education. Journal of Human Nutrition and Dietetics, 6: 323-333. doi:10.1111/j.1365277X.1993.tb00378. x

Towers, A. L., Burgio, K. L., \& Locher, J. L. (1994). Constipation in the elderly: influence of dietary, psychological and physiological factors. The Journal of American Geriatrics Society, 42, 701-706.

Wald, A., Scarpignato, C., \& Kamm, M. A. (2007). The burden of constipation on quality of life: results of a multinational survey. Alimentary Pharmacology and Therapeutic, 26, 227-235.

World Health Organization. (1993). Standard acupuncture nomenclature, second edition. A brief explanation of 361 classical acupuncture point names and their multilingual comparative list. Retrieved from: http://apps.who.int/medicinedocs/documents/s7143e/s7143e.pdf

Wu, H. S., \& Lin, L. S. (2003). Application of acupoint pressure on patients living with chronic breathing difficulties (in Chinese). Journal of Nursing, 50(1), 75-79. 
Yan, X. K., Chui, J. M., \& Yang, Y. Q. (2005). Development on clinical treatment of constipation with acupuncture and moxibustion. World Journal of AcupunctureMoxibustion, 15, 56-64.

Yoon, Y. S., Park, K. O., Kang, M. S., \& Ha, J. Y. (2014). Effect of abdominal massage and meridian acupressure on constipation in long-term care residents. Global Health \& Nursing, 4(1), 1-10.

Zhang, H. Z., Liu, X. M., Li, Y. C., Chen, X. F., \& Zhou, P. X. (2006). Literature reviews on the clinical application of acupoint pressure (in Chinese). The Journal of Long Term Nursing, 9(3), 91-101.

Zhang, L. Q., Dai, Y. C., \& Weng, Z. W. (2001). Risk factors of the older inpatients living with constipation. Taiwan Medicine, 5(6), 607-614. 
Table 1. Distribution of the institutions of the samples between the groups $(n=90)$

\begin{tabular}{llcc}
\hline Groups & Intervention & Site 1 & Site 2 \\
\hline Experimental group I & laxatives + abdominal massage & 22 & 8 \\
Experimental group II & laxatives + abdominal massage + APT & 23 & 5 \\
Control group & laxatives only & 22 & 10 \\
\hline
\end{tabular}

Note. $\mathrm{APT}=$ acupoint pressure therapy 
Table 2. Baseline comparison between the groups $(n=90)$

\begin{tabular}{|c|c|c|c|c|c|}
\hline & $\begin{array}{c}\text { Control } \\
\text { Group }(n=30)\end{array}$ & $\begin{array}{l}\text { Group I } \\
(\mathrm{n}=28)\end{array}$ & $\begin{array}{l}\text { Group II } \\
(\mathrm{n}=32)\end{array}$ & & \\
\hline & $\mathrm{n}(\%) /$ & $\mathrm{n}(\%) /$ & $\mathrm{n}(\%) /$ & & \\
\hline Variables & $\mathrm{M} \pm \mathrm{SD}$ & $\mathrm{M} \pm \mathrm{SD}$ & $\mathrm{M} \pm \mathrm{SD}$ & $\chi^{2} / F$ & $p$-value \\
\hline Age & $74.63 \pm 8.91$ & $78.87 \pm 8.86$ & $76.97 \pm 8.99$ & 1.70 & 0.19 \\
\hline Gender (male) & $16(53.3)$ & $16(57.1)$ & $19(59.4)$ & 0.81 & 0.66 \\
\hline $\begin{array}{l}\text { Presence of chronic } \\
\text { diseases (yes) }\end{array}$ & $19(63.3)$ & $18(64.3)$ & $26(81.3)$ & & 0.02 \\
\hline $\begin{array}{l}\text { Dependent on other for } \\
\text { activity (yes) }\end{array}$ & $27(90.0)$ & $24(86.7)$ & $27(90.0)$ & & 0.89 \\
\hline Bedridden (yes) & $21(70.0)$ & $24(85.7)$ & $22(68.8)$ & 0.82 & 0.66 \\
\hline $\begin{array}{l}\text { Open bowls < } 2 \\
\text { times/week (yes) }\end{array}$ & $8(26.7)$ & $8(28.6)$ & $(37.5)$ & 1.67 & 0.43 \\
\hline \multicolumn{6}{|l|}{ Methods of oral intake } \\
\hline NG tube & $10(33.3)$ & $4(14.3)$ & $7(21.9)$ & 4.14 & 0.13 \\
\hline Mouth & $20(66.7)$ & $24(85.7)$ & $25(78.1)$ & & \\
\hline Vegetable intake & & & & 3.14 & 0.18 \\
\hline 0 serves /day & & $2(7.1)$ & $3(9.4)$ & & \\
\hline 1 serves /day & & $2(7.1)$ & $3(9.4)$ & & \\
\hline 2 serves /day & $3(10.0)$ & $4(14.3)$ & $7(21.9)$ & & \\
\hline 3 serves & $15(50.0)$ & $20(71.5)$ & $9(59.3)$ & & \\
\hline Fruit intak & & & & 11.52 & 0.02 \\
\hline 0 serves & 8 & 2 & 5 & & \\
\hline 1 serves /day & 9 & 6 & 14 & & \\
\hline 2 serves /day & 14 & 20 & 13 & & \\
\hline Fluid intake ( $\mathrm{ml}$ / day) & $1280 \pm 1183$ & $495 \pm 174$ & $570 \pm 430$ & 15.74 & $<0.001$ \\
\hline
\end{tabular}

Note. $\mathrm{SD}=$ standard deviation; $\mathrm{NG}=$ nasogastric 
Table 3. The GEE models for the comparison of effective of acupoint pressure therapy and the frequency of defecation after controlling the interference factors between the groups $(\mathrm{n}=90)$

\begin{tabular}{|c|c|c|c|c|}
\hline Variables & Coefficients & SE & $Z$ & $p$-value \\
\hline \multicolumn{5}{|l|}{ Frequency of defecation } \\
\hline Intercept & 0.42 & 0.27 & 1.55 & 0.04 \\
\hline Experiment group II & 0.07 & 0.20 & 0.35 & 0.01 \\
\hline Experiment group I & -0.06 & 0.22 & $-0.27<$ & 0.79 \\
\hline Frequency of defecation & 0.02 & 0.01 & 1.33 & 0.18 \\
\hline Experiment group II / frequency of defecation & 0.02 & 0.02 & 0.95 & 0.34 \\
\hline Experiment group I / frequency of defecation & 0.00 & 0.02 & & 0.80 \\
\hline Presence of chronic disease & 0.02 & 0.1 & 0.13 & 0.90 \\
\hline Fluid intake (mL/day) & -0.00 & & -0.09 & 0.93 \\
\hline Quantity of fruit intake ( $1 / 0$ serves) & -0.03 & & -0.23 & 0.82 \\
\hline Quantity of fruit intake ( $2 / 0$ serves) & & 0.15 & 2.00 & 0.04 \\
\hline \multicolumn{5}{|l|}{ Stool nature } \\
\hline Intercept & 2.1 & 0.68 & 3.09 & $<0.001$ \\
\hline Experiment group II & -0.88 & 0.33 & 3.09 & $<0.001$ \\
\hline Experiment group I & -1.73 & 0.33 & -5.27 & $<0.001$ \\
\hline Stool nature & -0.04 & 0.03 & -1.30 & 0.19 \\
\hline Experiment group II / frequency of defecation & 0.05 & 0.04 & 1.31 & 0.19 \\
\hline Experiment group I /frequency of defecation & 0.06 & 0.04 & 1.61 & 0.11 \\
\hline Presence of chronic disease & 0.30 & 0.20 & 1.51 & 0.13 \\
\hline Fluid intake (mL/day) & 0.00 & 0.00 & 2.37 & 0.05 \\
\hline Quantity of fruit intake (1/0 serves) & 0.42 & 0.49 & 0.87 & 0.39 \\
\hline Quantity of fruit intake ( $2 / 0$ serves) & 0.72 & 0.45 & 1.62 & 0.01 \\
\hline
\end{tabular}

Note. $\mathrm{SE}=$ standard error 